\title{
Utility of a Relatively Affordable In-House HIV-1 Genotyping Assay for Drug Resistance Testing among Non B HIV-1 Infected Drug Naive Patients in Nigeria
}

\author{
J. A. Anejo-Okopi1,2*, H. Onywera ${ }^{3}$, I. O. Abah" ${ }^{4}$, A. O. Ebonyi ${ }^{2,5}$, 0. 0. Agbaji ${ }^{2,6}$, A. P. Agaba ${ }^{2,7}$, \\ S. Oguche ${ }^{2,5}$, O. S. Olonitola ${ }^{8}$, J. A. Idoko ${ }^{2,6}$, \\ ${ }^{1}$ Department of Microbiology, University of Jos, Jos, Nigeria \\ ${ }^{2}$ AIDS Prevention Initiative in Nigeria, Jos University Teaching Hospital, Jos, Nigeria \\ ${ }^{3}$ HIV-Research Laboratory, Kenya Medical Research Institute, Kisumu, Kenya \\ ${ }^{4}$ Department of Pharmacy, Jos University Teaching Hospital, Jos, Nigeria \\ ${ }^{5}$ Department of Pediatrics, University of Jos, Jos, Nigeria, \\ ${ }^{6}$ Department Medicine, University of Jos, Jos, Nigeria \\ ${ }^{7}$ Department Family Medicine, University of Jos, Jos, Nigeria \\ ${ }^{8}$ Department of Microbiology, Ahmadu Bello University, Zaria, Nigeria \\ Email: *josephokopi@yahoo.com
}

How to cite this paper: Anejo-Okopi, J.A., Onywera, H., Abah, I.O., Ebonyi, A.O., Agbaji, O.O., Agaba, A.P., Oguche, S., Olonitola, O.S. and Idoko, J.A. (2018) Utility of a Relatively Affordable In-House HIV-1 Genotyping Assay for Drug Resistance Testing among Non B HIV-1 Infected Drug Naive Patients in Nigeria. Advances in Microbiology, 8, 355-365. https://doi.org/10.4236/aim.2018.85023

Received: April 6, 2018

Accepted: May 27, 2018

Published: May 30, 2018

Copyright ( $) 2018$ by authors and Scientific Research Publishing Inc. This work is licensed under the Creative Commons Attribution International License (CC BY 4.0).

http://creativecommons.org/licenses/by/4.0/

(c) (7) Open Access

\begin{abstract}
Background: The introduction of antiretroviral (ARV) in resource-limited settings has increased life expectancy among non-B HIV-1 infected individuals. We used a validated In-house genotyping assay to characterize non-B HIV-1 and to determine drug resistance mutations among treatment-naive patients. Methods: Plasma samples from 105 HIV-1 infected drug-naive adult patients attending a tertiary hospital Jos, Nigeria were subjected to HIV-1 RNA extraction, reverse transcription amplification, and population-based sequencing of the partial $\mathrm{pol}$ gene on the ABI 3130xl genetic analyzer. Subtyping and phylogenetic analyses were performed by REGA Subtyping Tool v2.0 and MEGA v5.0 respectively. Drug resistance profiles were evaluated according to IAS-USA 2013 drug resistance mutations list. Result: One hundred samples (95.2\%) were successfully genotyped. The distribution of the non-B HIV-1 subtypes were; CRF02_AG-48\%, G-41.0\%, CRF06_cpx-6.0\%, and A-5.0\%. Ten percent of the isolates had at least one major drug resistance mutation in the $\mathrm{pol}$ gene. The drug-class specific resistance prevalences were $6.0 \%$ for NRTIs; M41L-1.0\%, K65KR-1.0\%, M184IM-1.0\%, M184V-2.0\%, and T215ADNT-1\%, 8.0\% for NNRTIs; K103N-2\%, 1.0\% for K101E, E138A, G190A, P225HP, Y181I, Y188L, Y181C including protease inhibitors' Q58E
\end{abstract}


(1.0\%). Conclusion: HIV-1 was heterogeneously distributed; CRF02_AG and G predominate and some known major mutations associated with NRTIs and NNRTIs were determined. The In-house assay is suitable for both characterization of non-B HIV-1 subtypes and detection of drug resistance at a significant lower cost than available commercial genotyping assays. This finding underscores the need to consider use of low-cost In-house genotyping assay as an alternative in resource-limited settings with non-B HIV-1 epidemic.

\section{Keywords}

HIV-1, Antiretroviral Therapy, Drug Resistance, Genotyping, Affordable, In-House

\section{Introduction}

Human Immunodeficiency Virus type 1 (HIV-1) is responsible for chronic infection leading to Acquired Immunodeficiency Syndrome (AIDS) as a result of chronic infection damage [1]. The clinical course of HIV-1 infection is due to a high variability of the viral strain. This heterogeneity is a result of the high frequency of viral replication errors; retroviral reverse transcriptase (RT) lacking proofreading functions. As the virus replicates, genetic variations produced has ability for continued viral survival and growth as well as resistance to antiretroviral drugs (ARVs). However, while ARV is reducing mortality rates from AIDS-related causes, the widespread and long-term usage of drugs raises concerns with regards to emergence of ARV associated resistance mutations in evolving HIV strains. As evidence on the impact of large scale treatment programs in reducing incidence and increasing life expectancy in developing countries continues to accumulate, efforts to increase wider use ARVs will be intensified [2] [3]. The use of potent ARVs can result in a profound and durable suppression of HIV-1 replication resulting in plasma HIV-1 viral load below levels of detection. The presence of some viral mutations introduced into the HIV-1 genome during replication can compromise efficacy, while others display susceptibility of the virus infectivity in combination with ARVs resistance. With this expected long-term and success of ART in developing countries, the coverage and programmatic effort should be complemented by HIV drug resistance (HIVDR) monitoring programs to ensure good clinical outcomes, and to reduce treatment failure and transmission of drug resistance [4]. Thus, there is a need for more and affordable genotyping assays in attempt to effectively monitor and control drug resistance as more individuals are initiated onto ART as recommended by World Health Organization [5]. In-house genotypic drug resistance testing has been successfully used in developed countries; for surveillance and monitoring of drug resistance in HIV infected individuals receiving ART, but has not been integrated into the continuum of care in most settings in Africa. Most international guidelines recommend drug resistance testing for adult or 
pediatric patients failing ART-first-line and second-line [6] [7]. However, the affordability due to high-cost of the technology, and infrastructure requirements have limited the implementation of similar approaches to utility of drug resistance monitoring in resource-limited-settings (RLS).

The Nigeria HIV treatment and monitoring guidelines do not currently recommend the use of drug resistance testing in clinical choice of ART for individuals failing first-line regimens [8] but are switched based primarily on clinical and virological (HIV-1 viral load) parameters. It is evident that incorporating drug resistance testing into treatment guidelines would be potentially cost effective in the near future. Considering the high cost of alternative second-line ARVs to available first-line, the use of drug resistance testing to identify patients who needs switching will conserve treatment options, minimize economic loss to individuals, program and the nation. Therefore, it is important to reduce the cost of drug resistance monitoring methods by using affordable In-house genotyping assay in order to improve access, quality of care and better treatment outcomes. The available In-house genotyping assay is designed for non-B subtypes with high sensitivity by detecting amino acid substitution at rare positions not commonly found in subtype B specific commercially available assays. The assay which is cost effective and affordable has similar designed protocol to the commercially available assays: HIV Ribonucleic RNA extraction, Reverse Transcription (RT) and nested Polymerase Chain Reaction (PCR) amplification of viral targets, Cycle sequencing using Big-Dye terminator and Bioinformatics methods for analysis of chromatograms, manually check the alignment for misaligned sequences, deletions and insertions and edit accordingly. It also involves the construction of phylogenetic tree using relevant tools to examine and review evolutional relationships and those with short branches for possible contamination [9] [10].

The In-house HIV-1 genotyping assay is sensitive for detection and sequencing of non-B HIV strain variants specifically and it is CDC-supported Genotyping assays that well popular in East African countries [11] which is currently being deplored to CDC supported Laboratories in Nigeria, though not yet widely in use for clinical decisions. There is the need for Nigeria Government to buy into this program and fund it for effective utilization of the available ARVs to reduce emergence and transmission of drug resistance mutations, and associated economic cost. The utility of this assay will not only support researchers, but also impact positively on the success of HIV treatment populations and stop the spread of HIV and ARV-resistant strains. This study aimed to describe frequency of DRMs in HIV-1 genotypes that circulate in drug naive patients, using a cost-effective in-house HIV-1 GRT suitable for non-B HIV-1 variants.

\section{Methods}

This study was carried out at the AIDS Prevention Initiative in Nigeria (APIN) CDC supported HIV clinic at the Jos University Teaching Hospital (JUTH), Jos. 
The entry point for all patients was either through HIV counseling and testing (HCT) or referrals from other services within Jos metropolis and neighboring states. One hundred and five (105) HIV-1 infected treatment-naive patients were recruited sequentially after obtaining informed consent between October 2010 and April 2011. The institutional review board at Jos University Teaching Hospital approved the study protocol. A structured questionnaire was used to collect basic demographic data from each participant with no previous ARV exposure and was aged $\geq 18$ years. Blood samples were collected in EDTA containers and plasma was extracted and cryopreserved. The samples were shipped in ice-parked containers to the Kenya Medical Research Institute HIV-Resistance Laboratory, Kisian Kisumu, where genotypic testing using affordable In-house genotyping System was done. Of 105 samples tested, 100 successfully amplified for genotypic drug resistance testing.

\subsection{Isolation and Amplification of HIV-1 Partial Pol Gene by RT-PCR and Nested PCR}

We use two amplification protocols for HIV pol gene as previously described [9], HIV viral RNA were extracted from plasma using the QIAamp Viral RNA mini kit (Qiagen, Hilden, Germany) followed by reverse-transcription of the RNA into cDNA using the outer primers Prt-F1-forward (2253 - 2275 nucleotides, nt) and RT-R1 reverse (nt 3370-3348) for RT-PCR. The cDNA were amplified by nested-PCR with primer Prt-F2 (forward, 2265 - $2288 \mathrm{nt}$ ) and RT - R2 (reverse, 3326 - $3304 \mathrm{nt}$ ). The amplified DNA fragments from nested-PCR were verified by visually comparing the intensity of each sample's band to that of the DNA mass ladder's bands of known DNA quantity for expected size by electrophoresis in $1.0 \%$ agarose gel stained with $0.5 \mu \mathrm{g} / \mathrm{ml}$ ethidium bromide and photographed under ultraviolet illumination. The fragments were purified using the QIA quick PCR purification kit (Qiagen, Hilden Germany) in spin columns, and direct population-based sequencing performed on both strands using Big Dye ${ }^{\circledR}$ Terminator v3.1 Cycle Sequencing kit on ABI 3130X1 Genetic Analyzer.

\subsection{Sequence Analyses, Determination of Drug Resistance Mutation and Subtyping and Phylogenetic Analyses}

The generated nucleotide sequences were viewed using the Sequence Analysis Software v3.7, and aligned and edited using Sequencher version 5.0, which assembles the six overlapping sequence segments for the six primers to form a contiguous sequence. Sequences with frame shifts or stop codons were excluded from analysis. The quality of the generated sequences was checked using Sequence Quality Assessment Tool (SQUAT). The sequences in fasta format were then subjected to the Stanford HIVdb algorithm (http://hivdb.stanford.edu/) for subtyping and determination of mutations conferring various antiretroviral drugs resistances. Mutations in the sequences were defined as differences from the consensus B reference sequence and were further characterized as RTI and 
PI associated resistance mutations. HIV-1 subtyping was performed using REGA HIV-1 subtyping tool V2.0 from Stanford HIV drug resistance database (http://hivdb.stanford.edu/), a worldwide subtype references were obtained from Los Alamos database (http://hiv-web.lanl.gov), and sequences were aligned against the known reference strains. Phylogenetic analysis was performed by the neighbor-joining (NJ) method as implemented in MEGA V.0. The boots canning method was used to detect and study recombination, as implemented in the SIMPLOT software v.2.5). The recombination was further confirmed with Recombination Identification Program (RIP) v.3.0 available online

(http://www.hiv.lanl.gov/content/sequence/RIP/RIP.html). Subtypes assignments by this method are shown to the right of the tree with isolates clustered around the various references. Analyze the sequence using the HIVDB program at http://hivdb.stanford.edu.

\section{Result}

Of 105 samples, the proportion of female and male was 55\% and $45 \%$ respectively. Mode of transmission; $98 \%$ were heterosexual and $2 \%$ blood transfusion, marital status; $69 \%$ married, 19\% single (never married), while $8 \%$ were separated/divorced and $5 \%$ were widowed. The median baseline CD4 cell count was 186 cells $/ \mathrm{mm}^{3}$ (IQR: 12 - 737). WHO disease staging of the subjects was: stage I (24\%) stage II (21\%), stage III (36\%) and stage IV (19\%). 50\% of the spouses of the participants were HIV positive, while $50 \%$ were HIV negative. The participants whose spouses were on ARV were 14\%, and those whose spouses were not on ARV were $86 \%$.

\subsection{Subtyping and Phylogenetic Analyses}

Subtyping and phylogenetic analyses were performed by REGA Subtyping Tool v2.0 and MEGA v6.0 respectively. Figure 1 showed the distribution relationship of different genetic subtypes in the pol region using 21 references from Los Alamos HIV Sequence database. The subtypes AG and G clustered around references from Nigeria and other West and Central African neighboring regions. Of 105 samples, 100 (95.2\%) were successfully amplified and Sequenced. The distribution of the non-B HIV-1 subtypes were; CRF02_AG (48\%), G (41.0\%), CRF06_cpx (6.0\%), and A1 (5.0\%). Ten percent (10.0\%) of the isolates had at least one major drug resistance mutation in the pol gene. We observed more major mutation in patients with subtype AG (4/10), G (4/10), A1 (1/10) and cpx06 (1/10). Drug resistance profiles were evaluated according to IAS-USA 2017 update of drug resistance mutations list [12].

\subsection{Detection of Major Drug Resistance, Accessory Mutations and Natural Polymorphisms}

The In-house genotyping assay results from the samples of all treatment naive revealed some major IAS-defined major DRM in the viral pol gene [12]. The 


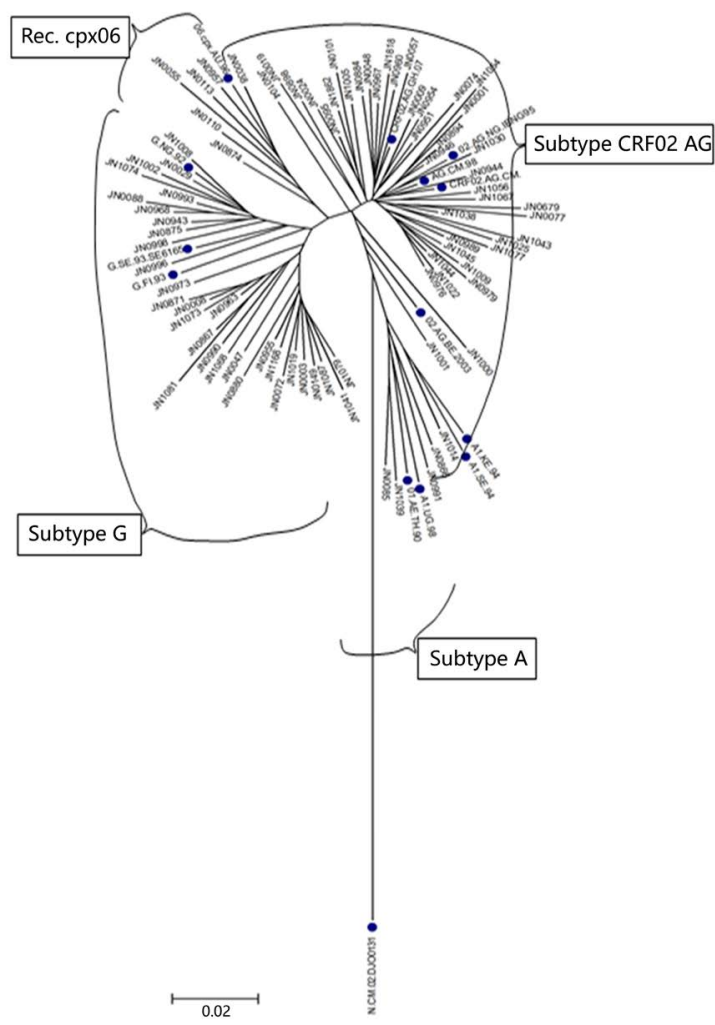

Figure 1. Phylogenetic Analysis of Isolates among the ARV Treatment naive Patients Jos, Nigeria.

frequency of the major DRMs for NRTIs, nNRTIs, and PIs among treatment-naive patients samples of subtype non-B are presented in Table 1. In the RT region, thymidine analogue mutations (TAMs) identified was M41L which could confer reduced susceptibility to currently approved nRTIs (AZT, d4T, ddI, $\mathrm{ABC}$ and TDF), however, the degree of cross-resistance depends on the number and presence of some accessory mutations. NRTIs (5.0\%), M41L (1.0\%), M184I (1.0\%), T215ADNT (1\%), K65KR-1.0\%, M184V (2.0\%).The presence of M184V prevent emergence of and accumulation TAMS of and other mutations. We observed nNRTIs (9.0\%), K103N (2\%), G190A (1.0\%), Y181I (1.0\%), Y181C (1.0\%), K101E (1.0\%), P225HP (1.0\%), Y188L (1.0\%), E138A (1.0\%), and PI major mutation was Q58E (1.0\%). Mutations K101E and E138A which occur as natural polymorphism and have been shown have reduced susceptibility to rilpirine. The TAMM41L was prevalent in patients with subtype AG (1.0\%). We identified T215 revertants in one patient in subtype A1 which may increase risk of developing virological failure on AZT- or d4T-containing first line ARVs because in contrast to the wild type threonine $(\mathrm{T})$, most revertants require just a single base-pair change to develop $\mathrm{T} 215 \mathrm{Y} / \mathrm{F}$ and because the presence of a revertant by standard sequencing may indicate the presence of $\mathrm{T} 215 \mathrm{Y} / \mathrm{F}$ as a minority variant [12]. K65R (1.0\%) was detected in one patient who also had nNRTI along Y181C. The frequency of the accessory mutations in the RT region were; R211K, V179IE, G196EG, V118I, V106I, A98SG, T69N, L228HL, and E44DE, 
Table 1. Prevalence of major ARV Resistance Mutations among the ARV Treatment-naive Patients.

\begin{tabular}{|c|c|c|c|c|c|c|c|c|c|c|}
\hline PTID & Age & Sex & $\begin{array}{l}\text { CD4+Cell } \\
\text { count }\end{array}$ & $\begin{array}{l}\text { HIV-1 } \\
\text { RNA VL }\end{array}$ & $\begin{array}{c}\text { Duration HIV-1 } \\
\text { Infection(AI) }\end{array}$ & $\begin{array}{l}\text { HIV-1 } \\
\text { Subtypes }\end{array}$ & $\begin{array}{l}\text { Spouse on } \\
\text { ART }\end{array}$ & \multicolumn{2}{|c|}{$\begin{array}{l}\text { Major RT mutations } \\
\text { NRT In NRTI }\end{array}$} & Major PR \\
\hline JN1073 & 34 & $\mathrm{~F}$ & 600 & 10,946 & Recent & G & No & - & - & Q58E \\
\hline JN1041 & 36 & $\mathrm{~F}$ & 18 & 23,050 & LI & G & Yes & M184I & $\begin{array}{l}\text { K101E, } \\
\text { Y181I }\end{array}$ & - \\
\hline JN0055 & 31 & $\mathrm{~F}$ & 31 & 99,892 & Recent & CRF06_cpx & No & - & Y188L & - \\
\hline JN0679 & 34 & M & 93 & 216,034 & LI & CRF02_AG & No & K65R & Y181C & - \\
\hline JN0866 & 27 & $\mathrm{~F}$ & 21 & 635,542 & LI & $\mathrm{A} 1$ & Yes & T215ADNT & - & - \\
\hline JN0990 & 39 & $\mathrm{~F}$ & 272 & 22,193 & LI & G & Yes & M184V & $\begin{array}{l}\text { K103N, } \\
\text { P225HP }\end{array}$ & - \\
\hline JN0088 & 45 & $\mathrm{~F}$ & 389 & 5788 & LI & G & Yes & M184V & $\begin{array}{l}\text { Y181C, } \\
\text { G190A }\end{array}$ & - \\
\hline JN0110 & 29 & $\mathrm{~F}$ & 237 & 99,898 & LI & CRF02_AG & No & M41L & - & - \\
\hline JN0114 & 43 & $\mathrm{~F}$ & 21 & 19,331 & LI & CRF02_AG & No & - & K103N & - \\
\hline JN1054 & 55 & M & 259 & 3758 & LI & CRF02_AG & No & - & E138A & - \\
\hline
\end{tabular}

V90I, and L210M.We also observed diverse natural polymorphisms which frequent in the protease gene as earlier reported L10I/V, V11LV, T12K,I13V, K14R, I15V, K20I/R/M , L33F, E34A M36I, N37DN, L38I, P39G/S,R41K, K45R, R57E/K, K61N, I62V, L63H,C67/G/S, H69K/Q/R, I72AEK/M/V/T, V82I L89M,, and the presence of an unusual mutation at position L90V (1\%); where $\mathrm{V}$ (valine) is observed instead of the M (methionine) non-B HIV-1 polymorphisms with various proportions [9].

\section{Discussion}

With the widespread availability of HAART in RLSs, the demand for sensitive and affordable monitoring of patients on ART is becoming a high priority. However, a better access to ARVs without adequate monitoring might result in a widespread transmission of drug resistant strains which will result to therapeutic failure. The current epidemic of drug-resistant HIV strains in RLSs can only be reduced by deliberate interventional strategies that would guarantee effective patients' regimen options, enhance correct intake of the medication, support uninterrupted drug supply, ensure the drug pharmaco-vigilance, treatment outcomes and provide useful therapeutic guidelines in case of treatment failures. The access to commercial genotypic assays and viral load monitoring in Africa is still limited and beyond the reach of the average patients due to high cost. Although these approved assays has demonstrated good performance on both subtype B non-B HIV-1, the high cost implication hinder their routine application in RLSs [13].

This study used a modified In-house GRT which had improved primer binding quality suitable for testing, the increased sensitivity, and found high degree of HIV-1 subtypes with the detection of diverse and rare mutations. The sub- 
types and drug resistance mutations identified corroborates with earlier reported findings in Africa including Nigeria [4] [14] [15] [16] [17] [18]. However, our finding of accessory mutations and polymorphisms both at RT and PR were more diverse and this could due to the broad sensitivity of the in-house genotyping assay used. Though the procedure without doubt still requires a well-equipped laboratory, but the assay can be performed for reagents cost of about 100 USD, which is significantly lower than the FDA approved available systems. Studies have reported high sensitivity of RT-PCR step with a detection limit of $<500$ copies/ml which permits its use as ascreening test in patients under treatment [19] [20]. The use of this method in the follow-up of patients under treatment will allow the fast detection of emerging drug resistance at a more affordable cost than the commercial tests. The in-house assay is broadly sensitive in the detection of drug resistance genotyping of HIV-1 group M viral strains and has proofed to be more sensitive than the, commercially available assays in detecting mixed viral populations (Zhou et al. 2011). The substantial reagent cost saving and broad sensitivity make this assay more accessible for RLS where HIV drug resistance surveillance is strongly recommended to minimize the development and transmission of HIV drug resistant mutants [21] [22] [23] [24].

Although recombinant subtype AG is still the most prevalent, it is responsible for only less than half of all the infections in Nigeria [9]. Studies have shown the extensive genetic diversity of HIV-1 strains from West Africa including Nigeria and Cameroon with significant representations of the recombinant form CRF02_AG, subtypes G, J, and F [25]. Although the performance of the two available genotyping assays can be considered as appropriate, but problems with non-B subtype strains do occur occasionally [21].

The main objective of this report was to present the utility of relatively affordable in-house HIV genotyping system for the monitoring of HIV-infected patients under treatment and surveillance. The method identified diverse major, accessory mutations and polymorphism which could be used as a guide for careful treatment options and further research into drug resistance surveillance, and the test can be performed at a lower cost compared to commercially available genotyping assays. Although, one is not ignorant of the need for expensive instrumentation, well trained personnel, the laboratory equipment requirement, however, the equipped laboratory can also be used for other applications including research. Now that the therapy has become widely available, affordable in-house genotyping for monitoring of patients on ARVs can be adopted and if properly utilized will avoid emergence of drug resistance in the population of HIV-1 strains.

\section{Conclusion}

HIV-1 was heterogeneously distributed; CRF02_AG and G predominate and some known major mutations associated with NRTIs, NNRTIs, diverse accessory, and natural polymorphism in the pol gene including one major mutation 
to $\mathrm{PIs}(\mathrm{Q} 58 \mathrm{E})$ were determined. The In-house HIV-1 genotyping assay is suitable for both characterization of non-B HIV-1 subtypes and detection of drug resistance at a significant lower cost than available commercial genotyping assays. This finding underscores the need to consider use of low-cost In-house genotyping assay as an alternative in resource-limited settings with non-B HIV-1 epidemic, and if well utilized, has potential to guide treatment options thereby keep the emergence of viral resistant strains at an acceptable level. In Nigeria, the burden of costs for treating HIV-infection is high, not only for the average citizens especially with the introduction of some laboratory investigation fees, but also the country's healthcare systems. Therefore the low cost and yet effective genotyping assay for non-B subtypes is a viable and practicable solution to expensive genotyping platforms.

\section{Acknowledgements}

We wish to acknowledge the CDC APIN Ltd GTE supported program for patients' care and management. We are also grateful to Jos University Teaching Hospital for permission to use the patients, and the management of HIV-Research Laboratory, Kenya Medical Research Institute, Kisumu, Kenya for permission to use the laboratory for the In-House HIV-1 genotyping assay.

\section{Funding}

This study was funded in part by the US Department of Health and Human Services, Health Resources and Services Administration (U51HA02522). The contents are solely the responsibility of the authors.

\section{Conflict of Interest}

The authors declare that they have no conflict of interest.

\section{References}

[1] Shao, Y. and Williamson, C. (2012) The HIV-1 Epidemic: Low- to Middle-Income Countries. Cold Spring Harbor Perspectives in Medicine, 2, a007187. https://doi.org/10.1101/cshperspect.a007187

[2] Tanser, F., Barnighausen, T., Grapsa, E., Zaidi, J. and Newell, M.L. (2013) High Coverage of ART Associated with Decline in Risk of HIV Acquisition in Rural Kwa Zulu-Natal, South Africa. Science, 339, 966-971. https://doi.org/10.1126/science.1228160

[3] Bor, J., Herbst, A.J., Newell, M.L. and Barnighausen, T. (2013) Increases in Adult Life Expectancy in Rural South Africa: Valuing the Scale-Up of HIV Treatment. Science, 339, 961-965. https://doi.org/10.1126/science.1230413

[4] Inzaule, S., Yang, C., Kasmebeli, A., Nafisa, L., Okonji, J., Oyaro, B., Lando, R., Mills, L.A., Laserson, K., Thomas, T., Nkengasong, J. and Zeh, C. (2013) Field Evaluation of a Broadly Sensitive HIV-1 In-House Genotyping Assay for Use with Both Plasma and Dried Blood Spot Specimens in a Resource-Limited Country. Journal of Clinical Microbiology, 51, 529. https://doi.org/10.1128/JCM.02347-12

[5] World Health Organization (2016) Guidelines on the Public Health Response to 
Pretreatment HIV Drug Resistance. Supplement to the 2016 Consolidated Guidelines on the Use of Antiretroviral Drugs for Treating and Preventing HIV Infection: Second Edition June 2016, 20 July 2017. World Health Organization, Geneva.

[6] Williams, I., Churchill, D., Anderson, J., Boffito, M., Bower, M., Cairns, G., Youle, M., et al. (2012) British HIV Association Guidelines for the Treatment of HIV-1-Positive Adults with Antiretroviral Therapy. HIV Medicine, 15, 1-85

[7] Onywera, H., Maman, D., Inzaule, S., Auma, E., Were, K., Zeh, C., et al. (2017) Surveillance of HIV-1 Pol Transmitted Drug Resistance in Acutely and Recently Infected Antiretroviral Drug-Naive Persons in Rural Western Kenya. PLoS One, 12, e0171124. https://doi.org/10.1371/journal.pone.0171124

[8] Anejo-Okopi, J.A., Onywera, H., Ebonyi, A., Agbaji, O.O., Agaba, A.P., Idoko, A.J., et al. (2014) High Frequency of Non-B HIV-1 Subtypes Specific Mutations at the Protease Gene among Treatment-Naive HIV-1 Infected Individuals in Jos, Nigeria. British Journal of Medicine and Medical Research, 4, 2303-2313. https://doi.org/10.9734/BJMMR/2014/7449

[9] Federal Ministry of Health (FMOH), Nigeria (2016) National Guidelines for HIV Prevention and Care in Adolescence and Adults. Federal Ministry of Health (FMOH), 60-62.

[10] Anejo-Okopi, J.A., Agbaji, O.O., Agaba, A.P., Harris, H., Were, K., Olonitola, O.S., et al. (2013) Human Immunodeficiency Virus Type-1 (HIV-1) Genetic Diversity and Prevalence of Antiretroviral Drug Resistance Mutations in Treatment-Naive Adults in Jos, North Central Nigeria. African Journal of Biotechnology, 12, 2279-2287. https://doi.org/10.5897/AJB2013.11954

[11] Wallis, L.C., Papathanasopoulos, A.M., Lakhi, S., Karita, E., Kamali, A., Stevens, W., et al. (2010) Affordable In-House Antiretroviral Drug Resistance Assay with Good Performance in Non-Subtype B HIV-1. Journal of Virological Methods, 63, 505-508. https://doi.org/10.1016/j.jviromet.2009.11.011

[12] Hassan, A.S., Nabwera, H.M., Mwaringa, S.M., Obonyo, C.A., Sanders, E.J., Berkley, J.A., et al. (2014) HIV-1 Virologic Failure and Acquired Drug Resistance among First-Line Antiretroviral Experienced Adults at a Rural HIV Clinic in Coastal Kenya: A Cross-Sectional Study. AIDS Research and Therapy, 11, 9. https://doi.org/10.1186/1742-6405-11-9

[13] Wensing, A.M., Calvez, V., Günthard, H.F., Johnson, V.A., Pillay, D., Shafer, R.W. and Richman, D.D. (2017) Update of the Drug Resistance Mutations in HIV-1. Topics in Antiviral Medicine, 24, 132-133.

[14] Ammaranond, P., Sanguansittianant, S., Raju, P.A., Cunningham, P. and Horthongkham, N. (2014) Development of a Cost-Effective Assay for Genotyping of HIV-1 Non-B Subtype for Drug Resistance. Journal of Virological Methods, 199, 102-107. https://doi.org/10.1016/j.jviromet.2014.01.007

[15] Ojesina, A.I., Sankalé, J.L., Odaibo, G., Langevin, S., Meloni, S.T., Kanki P.J., et al. (2006) Subtype-Specific Patterns in HIV Type 1 Reverse Transcriptase and Protease in Oyo State, Nigeria: Implications for Drug Resistance and Host Response. AIDS Research and Human Retroviruses, 22, 770-779. https://doi.org/10.1089/aid.2006.22.770

[16] Odaibo, G.N., Ola, S.O., Landerz, M., Dietrich, U. and Olaleye, D.O. (2012) HIV-1 Drug Resistant Mutations in Chronically Infected Treatment Naive Individuals in the Pre-ARV Era in Nigeria. African Journal of Medicine and Medical Sciences, 41, 61-63.

[17] Negedu-Momoh, O.R., Olonitola, O.S., Inabo, I. H., Inzaule, C.S., Oladele, A.E. and 
Agwale, M.S. (2014) Antiretroviral-Drug Resistant Mutations and Genetic Diversity in HIV-1 Infected Individuals in Nigeria. World Journal of AIDS, 4, 11.

[18] Agwale, S.M., Zeh, C., Robbins, K.E., Odama, L., Saekhou, A., Edubio, A., Njoku, M., Sani-Gwarzo, N., Gboun, M.S., Gao, F., Reitz, M., Hone, D., Pieniazek, D., Wambebe, C. and Kalish, M.L. (2002) Molecular Surveillance of HIV-1 Field Strains in Nigeria in Preparation for Vaccine Trials. Vaccine, 20, 2131-2139. https://doi.org/10.1016/S0264-410X(02)00059-2

[19] Lihana, R.W., Ssemwanga, D., Abimiku, A. and Ndembi, N. (2012) Update on HIV-1 Diversity in Africa: A Decade in Review. AIDS Reviews, 14, 83-100.

[20] Chew, K.K., Ng, K.Y., Khong, W.X., Kaur, P., Thoon, K.C., Ng, O.T., et al. (2012) Clinical Evaluation of an In-House Human Immunodeficiency Virus (HIV) Genotyping Assay for the Detection of Drug Resistance Mutations in HIV-1 Infected Patients in Singapore. Annals of the Academy of Medicine, Singapore, 41, 553-558.

[21] Yang, C., McNulty, A., Diallo, K., Zhang, J., Titanji, B., Nkengasong, J.N., et al. (2010) Development and Application of a Broadly Sensitive Dried-Blood-Spot-Based Genotyping Assay for Global Surveillance of HIV-1 Drug Resistance. Journal of Clinical Microbiology, 48, 3158-3164. https://doi.org/10.1128/JCM.00564-10

[22] Zhao, H., Liao, L., Xing, H., Wang, Z., Su, B., Shao, Y., et al. (2015) Evaluation of an In-House HIV-1 Drug Resistance Genotypic Testing for Using Dried Blood Spot Specimens in China. Current HIV Research, 13, 109-116. https://doi.org/10.2174/1570162X13666141210154647

[23] Zhou, Z., Wagar, N., DeVos, J.R., Rottinghaus, E., Nkengasong, J., Yang, C., et al. (2011) Optimization of a Low Cost and Broadly Sensitive Genotyping Assay for HIV-1 Drug Resistance Surveillance and Monitoring in Resource-Limited Settings. PLOS ONE, 11, e28184. https://doi.org/10.1371/journal.pone.0028184

[24] Chaturbhuj, D.N., Deshmukh, P.S., Hingankar, N.K., Siddhaarth, K., Deshpande, S.N., Tripathy, S.P., et al. (2013) Evaluations of an In-House Drug Resistance Method for HIV-1 Drug Resistance Using ViroSeq ${ }^{\text {Tm }} 2.0$ Genotyping System as a Gold Standard. Journal of Virological Methods, 189, 87-92. https://doi.org/10.1016/j.jviromet.2013.01.001

[25] Fonjungo, P.N., Mpoudi, E.N., Torimiro, J.N., Alemnji, G.A., Nkengasong, J.N., Pieniazek, D., et al. (2002) Human Immunodeficiency Virus Type 1 Group m Protease in Cameroon: Genetic Diversity and Protease Inhibitor Mutational Features. Journal of Clinical Microbiology, 40, 837-845.

https://doi.org/10.1128/JCM.40.3.837-845.2002 